\title{
Evaluación de las prácticas parentales: Análisis psicométrico de la escala Adolescent Family Process
}

\author{
Parental processes assessment: \\ Psychometric analysis of the Adolescent Family Process Scale
}

\author{
Antonella Bobbio * ${ }^{1}$, Karin Arbach ${ }^{1}$, Ana Alderete ${ }^{1}$ \\ 1 - Centro de Investigaciones de la Facultad de Psicología (CIPSI), Grupo Vinculado, \\ Centro de Investigaciones y Estudios sobre Cultura y Sociedad (CIECS), \\ Consejo Nacional de Investigaciones Cientificas y Técnicas (CONICET) Córdoba, Argentina.
}

\author{
Introducción \\ Método \\ Resultados \\ Discusión \\ Referencias
}

\section{Resumen}

Es en la adolescencia cuando se registra la mayor prevalencia de comportamientos antinormativos. Las prácticas parentales influyen de manera directa sobre el mantenimiento o desistencia de estas conductas en los hijos adolescentes. Este artículo explora las propiedades psicométricas de la escala Adolescent Family Process en un grupo de 301 adolescentes de Córdoba (Argentina), $27 \%$ de ellos con antecedentes delictivos. Esta herramienta se utiliza frecuentemente en estudios criminológicos para la medición de la relación entre las prácticas parentales y el comportamiento antinormativo de los adolescentes. De manera general, la escala que aquí se analiza resultó válida y fiable para el estudio de las prácticas parentales en esta población. Los índices de consistencia interna resultaron adecuados y los análisis de validez de criterio indicaron que distintos tipos de comportamiento antinormativo se asocian negativamente con prácticas parentales como el monitoreo, la comunicación y la aprobación, y positivamente con el conflicto entre padres e hijos. No obstante, los análisis factoriales exploratorios mediante ejes principales indicaron una estructura factorial de cinco factores diferente a la propuesta en la escala original.También las escalas de prácticas paternas y maternas mostraron diferencias en la estructura factorial. Se discuten las implicaciones teóricas y técnicas de estos hallazgos.

Palabras clave: prácticas parentales, adolescentes, análisis psicométrico, castellano

\begin{abstract}
The highest prevalence of antinormative behaviors occurs in adolescence. Parenting practices influence directly on the maintenance or desistance of these behaviors. This paper explores the psychometric properties of the Adolescent Family Process scale in a group of 301 teenagers from Cordoba (Argentine), $27 \%$ of them with criminal records. The scale is frequently used in criminological studies for the measurement of the relationship between parenting practices and the antinormative behavior of teenagers. In a general way, the scale proved to be valid and reliable for the study of parenting practices in this population. Internal consistency rates were adequate and criterion validity analysis showed that different types of antinormative behavior are negatively associated with parental practices such as monitoring, communication and approval, and positively correlated with conflict between parents and children. However, exploratory factor analysis (principal axes) indicated a five factorial structure different from those purposed in the original scale. The paternal and maternal practices scales showed differences in the factorial structure. Theoretical and technical implications of findings are discussed
\end{abstract}

Key words: parental practices, teenagers, psychometric analysis, Spanish

\footnotetext{
*Correspondencia a: Antonella Bobbio. Dirección Postal: Enfermera Gordillo esquina Enrique Barros, Facultad de Psicología (Laboratorio de Evaluación Psicológica y Evaluativa), Ciudad Universitaria, Córdoba, Argentina. Teléfono: +54 3519 2056764. abobbio@psyche.unc.edu.ar

Cómo citar este artículo: Bobbio, A; Arbach, K; \& Alderete, A. (2016). Evaluación de las prácticas parentales: Análisis psicométrico de la escala Adolescent Family Process. Evaluar, 16, 46-65. Recuperado de https://revistas.unc.edu.ar/index.php/revaluar
} 


\section{Introducción}

La familia es el primer entorno donde el niño adquiere habilidades y aprendizajes que le permitirán un adecuado ejercicio de su autonomía y de conductas necesarias para su desarrollo (Torío-López, 2001). Durante la adolescencia, la forma en que los padres interactúan con sus hijos ejerce una influencia decisiva en la forma en que estos avanzan hacia la adultez (Polo, 2009).

El estudio de las relaciones familiares $\mathrm{y}$, más específicamente, de la forma en que los padres ejercen su rol, ha ocupado un lugar muy importante en la literatura. Tradicionalmente una de las formas más empleadas en el abordaje de la función parental ha sido el estudio de los estilos educativos (Asili-Pierucci \& Pinzón-Luna, 2003; Baumrind, 2005; Maccoby \& Martin, 1983; McBride-Chang \& Chang, 1998; PonsDiez \& Berjano-Peirats, 1997). Los estilos educativos refieren a la forma en que se organizan patrones persistentes del comportamiento de los padres ante distintas formas de actuación de los hijos (Pérez-Alonso, 2012). A partir de la interacción entre dos dimensiones fundamentales, como son el afecto y el control, se han descrito cuatro estilos parentales: democrático, autoritario, permisivo e indiferente (Maccoby \& Martin, 1983). Los estilos educativos democráticos favorecerían un mayor ajuste psicosocial en los adolescentes, quienes presentarían menos problemas internalizantes (como ansiedad y depresión) y externalizantes (problemas de comportamiento, violencia) que los jóvenes que provienen de familias con otros estilos parentales (Milevsky, Schlechter, Netter, \& Keehn, 2007; Pelegrina-López,
García-Linares, \& Lendínez, 2002; Steinberg, 2001; Suldo \& Huebner, 2004).

Sin embargo, en los últimos años algunos investigadores han resaltado la necesidad de una descripción más flexible y dinámica de las conductas parentales (Metzler, Biglan, Ary, \& Li, 1998; Roberts \& Steinberg, 1999; Snider, Clements, \& Vazsonyi, 2004; Vazsonyi, Hibbert, \& Blake-Snider, 2003). Desde esta perspectiva se propone analizar el rol de los padres con un enfoque dimensional, en detrimento de una valoración tipológica (Molinero, 2006). Esto ha dado lugar a un estudio sistemático de lo que se ha denominado prácticas parentales. La diferencia principal entre ambas formas de aproximación a las dinámicas familiares es que, mientras el estilo parental es considerado como un patrón de respuestas o como un conjunto general de actitudes en el cual se enmarcan los comportamientos parentales, las prácticas son las conductas específicas de los padres en cada situación (Hernández-Guzmán, Montesinos, Bermúdez-Ornelas, Freyre, \& Alcázar-Olán, 2013). Algunos autores consideran que las prácticas parentales resultan de mayor utilidad que los estilos para predecir comportamientos en los hijos (Carlo, McGinley, Hayes, Batenhorst, \& Wilkinson, 2007).

La escala Adolescent Family Process (AFP): desarrollo y aplicación

A partir de una perspectiva dimensional Metzler et al. (1998) proponen la existencia de seis prácticas parentales: relaciones familiares positivas, uso de reforzamiento positivo, monitoreo parental, establecimiento de normas por parte de los padres, fortalecimiento consis- 
tente de las normas, y conflicto padre-hijo. Por su parte, Steinberg y Silk (2002) han postulado tres grandes dimensiones de prácticas parentales: armonía, autonomía y conflicto. La armonía representa la dimensión afectiva y la calidez que los padres tienen con sus hijos, mientras que la autonomía refiere al control, las restricciones, la supervisión y el apoyo que brindan los padres a sus niños (Steinberg \& Silk, 2002). El conflicto, por su parte, es la tensión normal entre padres e hijos que, dependiendo de la intensidad y de otras variables del entorno familiar, puede ser positivo o negativo para el desarrollo del menor (Roberts \& Steinberg, 1999).

Las formulaciones previamente descritas representan la base teórica a partir de la cual se desarrolló la escala Adolescent Family Process (AFP; Vazsonyi et al., 2003). Se trata de una herramienta de autoinforme para la evaluación de prácticas parentales reportadas por adolescentes. El instrumento permite valorar prácticas paternas y maternas de manera independiente, ya que, según indican los autores, la cantidad y calidad de tiempo que los padres pasan con sus hijos podría ser cuantitativa y cualitativamente diferente entre ambos sexos (Stephens, 2009; Videon, 2005). La AFP se compone de seis subescalas (cercanía, soporte, monitoreo, comunicación, conflicto $\mathrm{y}$ aprobación del grupo de pares) que, de manera general, representan las tres dimensiones propuestas previamente por Steinberg y Silk (2002): armonía (cercanía y comunicación), autonomía (soporte, aprobación del grupo de pares y monitoreo) y conflicto. La cercanía se refiere a la proximidad emocional y a la sensación de afecto y comprensión adecuados, mientras que la comunicación se refiere al intercambio de información de manera afectiva entre padres e hijos. Si bien originalmente esta subescala estaba dividida en dos tipos de comunicación (instrumental e ínti- ma), los análisis psicométricos indicaron que se trata de una misma dimensión (Vazsonyi et al., 2003). El soporte es definido como una actitud de apoyo, aceptación y aprobación por parte de los padres (Molinero, 2006). La subescala de monitoreo analiza las conductas de supervisión y el conocimiento por parte de los padres de las actividades que realizan sus hijos. La aprobación de pares hace referencia a la aceptación que los padres manifiestan respecto a los amigos que tienen sus hijos y se relaciona con la autonomía y con la confianza en sus propias elecciones (Ozdemir, Vazsonyi, \& Cok, 2013). Finalmente, el conflicto es entendido de manera similar a lo que señalan Roberts y Steinberg (1999), es decir, no como algo negativo en sí mismo, sino dependiente del nivel en que se manifiesta y de la interacción con otras variables familiares (Vazsonyi et al., 2003).

Estudios con jóvenes de diferentes países de América y Europa indican que la AFP posee buenas propiedades psicométricas tanto en el caso de las prácticas paternas como en el de las maternas (Pickering \& Vazsonyi, 2010; Snider et al., 2004; Vazsonyi \& Belliston, 2007; Vazsonyi et al., 2003; Vazsonyi, Ksinan-Jiskrova, Ksinan, \& Blatny, 2016). Así, la estructura factorial de seis dimensiones ha obtenido apoyo para su uso con adolescentes en Hungría, Suiza, Holanda y Estados Unidos (Vazsonyi et al., 2003). Los índices de consistencia interna de las subescalas han demostrado ser de moderados $(\alpha=.64)$ a buenos $(\alpha=.89)$ (Ozdemir et al., 2013; Pickering, Smith, \& Vaughn, 2005; Snider et al., 2004), y los análisis de validez externa han evidenciado fuertes asociaciones entre las prácticas parentales medidas con la AFP y variables teóricamente relacionadas a éstas, como la delincuencia juvenil (Vazsonyi et al., 2003).

Para analizar las propiedades psicométri- 
cas de la versión en castellano se llevó a cabo un estudio en 276 adolescentes de Perú (Molinero, 2006). Los resultados de los análisis factoriales indicaron las seis dimensiones parentales propuestas en la versión original. No obstante, se evidenció una importante presencia de cargas secundarias, esto indica que si bien los ítems representan los factores originalmente propuestos, también se relacionan significativamente con otros factores indicando una estructura factorial compleja y una elevada covariación entre los ítems.

Los índices de confiabilidad reportados para la versión española han sido de moderados a buenos (Ascenzo-Bravo de Rueda, 2012; Molinero, 2006), aunque inferiores a los encontrados en los trabajos que utilizan la versión original en inglés (Ozdemir et al., 2013; Pickering \& Vazsonyi, 2010; Snider et al., 2004; Vazsonyi \& Rudi, 2008).

Prácticas parentales y conducta antinormativa adolescente

El papel que los padres desempeñan en la crianza de los hijos es de gran importancia, tanto para promover un adecuado ajuste psicosocial como para evitar que se involucren en comportamientos riesgosos (Franco-Nerín, Pérez-Nieto, \& De Dios-Pérez, 2014; Oliva, Parra, \& Arranz, 2008). Si bien las prácticas parentales son de importancia en diferentes instancias del desarrollo, lo son aún más durante la adolescencia por ser una etapa de particular vulnerabilidad para la manifestación de ese tipo de comportamientos. Diversos estudios han demostrado una alta prevalencia de comportamientos antinormativos en este período vital (Arbach, Santuoro, Lumello, \& Garrido, 2013; Piquero, Hawkins, Kazemian, Petechuk, \& Redondo, 2013; Rechea, 2008). La manera en que los padres se comportan con sus hijos es una de las variables de mayor influencia en la conducta desviada de éstos (Hoeve et al., 2009, 2012; Loeber, 1990; Petrosino, Derzon, \& Lavenberg, 2009). Los investigadores han intentado discernir qué prácticas parentales se relacionan, y en qué medida, con la conducta delictiva de los hijos, ya que esto podría variar en función de la cultura y la población (Vazsonyi \& Flannery, 1997). Por ejemplo, una revisión de 161 artículos sobre parentalidad y delincuencia encontró que una serie de dimensiones negativas de la parentalidad como la negligencia, la hostilidad, la permisividad y el rechazo se asociaban positivamente al comportamiento delictivo de los hijos; mientras que el monitoreo parental, la comunicación y las dimensiones positivas del apoyo mantenían una asociación negativa con la delincuencia (Hoeve et al., 2009). Un estudio con jóvenes de Oklahoma también mostró que el monitoreo parental, el vínculo y la comunicación entre padres e hijos disminuyen el involucramiento en comportamientos violentos (Haegerich, Oman, Vesely, Aspy, \& Tolma, 2014). Otro trabajo con 306 adolescentes en riesgo social de Marruecos y Holanda indicó que la comunicación y el apoyo de los padres se relacionaban de manera negativa con la delincuencia de sus hijos, mientras que la autonomía y el conflicto no tuvieron una relación con la delincuencia en esta muestra (Eichelsheim et al., 2010). Un estudio español encontró que las prácticas parentales caracterizadas por hostilidad, irritabilidad y rechazo guardaban una relación positiva con el comportamiento agresivo en una muestra de 1.285 jóvenes españoles, mientras que una convivencia afectuosa, el apoyo emocional y la estimulación hacia la autonomía personal guardaban una relación negativa con dicha con- 
ducta (Mestre, Samper, \& Frías, 2004).

Más específicamente, las relaciones entre las dimensiones de la AFP y distintos tipos de conducta antinormativa han demostrado ser consistentes. Por ejemplo, estudios con adolescentes de Hungría, Holanda, Estados Unidos y Suiza encontraron que las dimensiones de la AFP se asociaban negativamente a distintos tipos de conducta antisocial (como el vandalismo, el hurto, las agresiones, el mal comportamiento escolar y el consumo de alcohol y drogas), con excepción del conflicto, que evidenciaba relaciones positivas (Vazsonyi, 2003; Vazsonyi et al., 2003). Otro estudio evidenció que la cercanía, el soporte y la aprobación de los pares estaban relacionados de forma negativa a la agresión en jóvenes de Turquía (Ozdemir et al., 2013). La cercanía, el monitoreo y el apoyo también evidenciaron asociaciones negativas con la conducta desviada en adolescentes suecos, incluyendo una amplia gama de comportamientos relacionados al consumo de sustancias (alcohol y drogas), al robo, al vandalismo y a la violencia (Vazsonyi \& Rudi, 2008).

Debido a esta fuerte vinculación entre las prácticas parentales y el comportamiento antisocial adolescente, los trabajos que valoran la validez predictiva de los instrumentos de evaluación de prácticas parentales suelen emplear medidas relacionadas a la delincuencia (Cernkovich \& Giordano, 1987) y al consumo de sustancias (Metzler et al., 1998) como variables criterio.

El presente estudio

En Argentina se han construido y validado distintos instrumentos para el estudio de las prácticas parentales (Richaud, 2007) o de los estilos educativos (Brussino \& Alderete, 2002; Sánchez, 2014) demostrando buenas propiedades psicométricas. No obstante, algunos sólo se han validado en niños (Brussino \& Alderete, 2002; Richaud, 2007) o evalúan el rol parental a través de los estilos parentales (Sánchez, 2014) y, como se ha señalado previamente, este trabajo ha optado por una perspectiva más dinámica. La AFP ha sido elegida porque cuenta con la particularidad de ser una herramienta sistemáticamente utilizada en criminología para el estudio de la relación entre prácticas parentales y conducta antinormativa en adolescentes. El presente estudio se enmarca en una línea de trabajo que se propone investigar los comportamientos delictivos juveniles de alta prevalencia en esta etapa de la vida, y para ello se cree indispensable contar con herramientas que hayan sido diseñadas, validadas y utilizadas en los contextos específicos de interés y que permitan una adecuada comparación transcultural. A su vez, la AFP ha demostrado ser válida y fiable en estudios que han empleado tanto la versión en inglés como la adaptación al castellano y ha sido construida en base a una perspectiva dimensional que permite una adecuada descripción de un variado número de comportamientos parentales. Es por esto que el presente trabajo se propuso estudiar las propiedades psicométricas de la AFP en adolescentes de Córdoba. Se evaluó su estructura factorial a través de un análisis factorial exploratorio y se analizó su consistencia interna mediante el estadístico alfa de Cronbach. También se valoró la validez de criterio a través de su asociación con diferentes tipos de conducta antinormativa. 
Variables e Instrumentos

\section{Método}

\section{Participantes}

La muestra fue no probabilística de tipo incidental y estuvo conformada por 301 jóvenes de ambos sexos (61\% varones, 39\% mujeres) con edades comprendidas entre los 12 y los 18 años $(\mathrm{M}=15.8 ; \mathrm{DE}=1.9)$ de la provincia de Córdoba (Argentina). Del total de participantes el 27.2\% eran jóvenes infractores residentes, al momento de la evaluación (abril a octubre de 2015), en uncentro socioeducativo cerrado de la ciudad de Córdoba. Los restantes eran alumnos de dos institutos educativos públicos de nivel medio radicados en dos localidades del interior de la provincia y pertenecientes a estratos socioeconómicos medios a bajos. La inclusión de participantes de diferentes grupos poblacionales (estudiantes e infractores de ley) tuvo por objetivo emplear una muestra heterogénea, donde los diferentes niveles de las variables en estudio estuvieran representados. El 50\% de adolescentes manifestó convivir con ambos padres, el $42 \%$ sólo convivía con uno de ellos y el $8 \%$ indicó no convivir con ninguna figura parental. En relación al máximo nivel educativo alcanzado por la madre, el 52\% reportó estudios secundarios o superiores completos y el $38 \%$ indicó primaria completa. Para el caso del padre, el 49\% manifestó que había terminado el secundario o los estudios superiores, mientras que el $38 \%$ dijo que su padre había completado los estudios primarios.
Prácticas parentales. Fueron valoradas con la versión en castellano (Molinero, 2006) de la escala Adolescent Family Process (Vazsonyi et al., 2003). La escala evalúa la percepción que tienen los adolescentes sobre las relaciones con sus madres y padres (biológicos, sustitutos o tutores). La escala se compone de seis subescalas: proximidad (seis ítems; p. ej., "mi mamá es cariñosa conmigo”), soporte (cuatro ítems; p. ej., "mi mamá a veces no me escucha y no tiene en cuenta mis opiniones"), monitoreo (cuatro ítems; p. ej., "cuando paso tiempo libre fuera de casa, mi mamá sabe dónde y con quién estoy”), comunicación (cinco ítems; p. ej. "le cuento a mi mamá sobre decisiones personales importantes"), conflicto (tres ítems; p. ej., "peleo bastante seguido con mi mamá") y aprobación del grupo de pares (tres ítems; p. ej., " a mi mamá en general le caen bien mis amigos"). Cada ítem se responde en una escala tipo Likert de cinco puntos $(0=$ "totalmente falso" a 4 = "totalmente cierto"). Se llevaron a cabo las mínimas adaptaciones necesarias para adecuar el vocabulario a la edad, contexto y nivel de instrucción de los participantes. Para ello se solicitó la colaboración de educadores y profesionales que trabajaban con los adolescentes y la supervisión de un experto en psicometría. Atendiendo a sus sugerencias, los ítems de las cuatro últimas subescalas de la AFP fueron reformulados de forma afirmativa para unificar los criterios de respuesta en todos los ítems de la escala y evitar que el cambio en la manera de responder pudiera generar confusión en los participantes. En el instrumento original estos ítems se formulan como preguntas con cinco categorías de respuesta diferentes a los otros factores, de "nunca" (0) a "muy frecuentemente" (4). De la misma manera que en la versión original, en la versión reformulada 
un puntaje más alto indica mayor presencia de la variable. Siguiendo la estrategia de los autores, los ítems de la subescala soporte se recodificaron a la inversa $(0=$ totalmente cierto a $4=$ totalmente falso), de manera que, como en el resto de las subescalas, un puntaje más alto indica más presencia de la variable.

Conducta antinormativa. Se valoró con la versión adaptada al castellano (Garrido, Cupani, \& Arbach, 2015) de la Escala de Conducta Antinormativa (NDS por su nombre en inglés: Normative Deviance Scale; Vazsonyi, Pickering, Junger, \& Hessing, 2001). Esta escala se compone de 47 ítems con respuesta tipo Likert de cinco puntos (desde $0=$ "nunca lo hice" a $4=$ "lo hice 6 veces o más”) que exploran un amplio espectro de actividades desviadas y conductas de violación de la norma que son independientes de las definiciones culturales de delito y desviación propias de cada contexto sociocultural. Hay suficiente evidencia de su fiabilidad en diferentes muestras $(\alpha$ $=.90$ - .99; Arbach et al., 2013; Vazsonyi \& Belliston, 2007; Vazsonyi et al., 2001). A los fines del presente trabajo se emplearon cinco de las siete subescalas del instrumento: uso de alcohol, uso de drogas, vandalismo, robo y agresiones físicas. La subescala de conducta desviada general no fue utilizada debido a que reúne ítems poco específicos en relación a la tipología delictiva, mientras que la subescala de mal comportamiento escolar no pudo ser evaluada debido a que un gran porcentaje de los menores infractores no estaban escolarizados (44\%). Los índices de fiabilidad de las NDS total y de las subescalas en la muestra aquí analizada fueron aceptables a excelentes ( $\alpha$ $=.71$ a $\alpha=.94)$.

\section{Procedimiento}

Luego de obtener las autorizaciones institucionales de los centros donde se encontraban los adolescentes, se procedió a la recogida de datos. Se solicitó a los participantes la firma del consentimiento informado ajustado a las normas éticas de cada institución. En el mismo se expresaban con claridad los objetivos del estudio, sus implicancias, el carácter voluntario de la participación y la confidencialidad en el tratamiento de los datos.

En el centro socioeducativo cerrado la aplicación se llevó a cabo de manera colectiva en grupos de dos a cinco sujetos en lugares dispuestos por la institución para tal fin. Cuando el participante carecía de habilidades lecto-comprensivas adecuadas para cumplimentar el protocolo de recogida de datos pero manifestaba interés en participar se administraba el instrumento de forma verbal e individualizada. Sin embargo, aunque la consigna y los ítems del instrumento eran verbalizados por el investigador, las respuestas en todos los casos fueron escritas en el protocolo por los participantes garantizando, de esta manera, el anonimato. En el caso de los centros educativos se administraron los instrumentos en las aulas de manera colectiva y ante la presencia de un docente y de la responsable de la investigación (A.B.).

\section{Análisis de datos}

Para el análisis de datos se empleó el paquete estadístico informatizado SPSS versión 22 (IBM Corp., 2013). 
Estructura Factorial. Se llevó a cabo un análisis factorial exploratorio (AFE) para cada escala (AFP madre y AFP padre). Se utilizó el método de extracción de ejes principales con rotación promax (oblicua) dado que ambas escalas presentaban algunas correlaciones interfactoriales superiores a .32 (Tabachnick \& Fidell, 2001). Se optó por un criterio teórico para la estimación de factores y se solicitó una solución de seis factores (tal como propone la escala original) para garantizar la interpretabilidad de la solución encontrada (Lloret-Segura, Ferreres-Traver, Hernández-Baeza, \& Tomás-Marco, 2014). En función del tamaño muestral se decidió eliminar los ítems que obtuvieran cargas factoriales inferiores a .35 o a aquellos que tuvieran cargas secundarias superiores a 32 (Hair, Anderson, Tatham, \& Black, 1999; Tabachnick \& Fidell, 2001).

Consistencia interna. Se evaluó la consistencia interna de las escalas totales y de cada una de sus subescalas mediante el estadístico alfa de Cronbach $(\alpha)$. Coeficientes superiores a .70 son considerados aceptables, mientras que aquellos que superan .80 y .90 se consideran buenos y excelentes, respectivamente. Los índices comprendidos entre .60 y .70 son considerados moderados, y se sugiere no aceptar valores inferiores a éstos (George \& Mallery, 2001).

Validez de criterio. Para evaluar la validez de criterio de las escalas, se examinaron las relaciones entre las dimensiones de éstas y distintos tipos de conducta antinormativa (consumo de alcohol, consumo de drogas, vandalismo, robo y agresiones físicas) medidos con la Escala de Conducta Antinomativa (NDS; Garrido et al., 2015; Vazsonyi \& Belliston, 2007). Las correlaciones fueron interpretadas según el criterio tradicion- al basado en rangos: de .0 a .25 la correlación es considerada escasa; de .26 a .50 débil; de .50 a .75 entre moderada y fuerte; y superior a .75 entre fuerte y perfecta (Santander-Montes \& Ruiz-Vaquero, 2004).

\section{Resultados}

Previo a la exploración de las propiedades psicométricas de la escala, se evaluó la calidad de la base de datos a través de un análisis exhaustivo de los ítems del instrumento. Del total de participantes 293 sujetos respondieron el cuestionario para la madre y 240 el del padre. Se eliminaron aquellos sujetos que dejaron más del $10 \%$ de cada escala sin completar (15 casos para el caso de la madre y 13 para el del padre). La distribución de valores perdidos fue aleatoria $\mathrm{y}$ no superó el $5 \%$ en ningún ítem, con excepción del ítem $\mathrm{n}^{\circ} 24$, “Le cae bien mi novia/o", cuyo porcentaje de valores perdidos estuvo en torno al $20 \%$ en las escalas de ambos padres. Esto se debe a que el contenido del ítem hace referencia a relaciones de pareja, y dada la corta edad de algunos participantes aún no han mantenido relaciones de este tipo y por eso no pudieron responder al ítem. No obstante, debido a que éste corresponde a una subescala conformada por sólo tres variables, se optó por mantenerlo en los análisis posteriores. Los ítems incompletos se trataron mediante imputación simple por la moda de la subescala a la que pertenecía ese ítem para cada sujeto. Esta estrategia fue elegida dado que la imputación por la moda ofrece equilibrio y simplicidad conceptual cuando se registran datos perdidos por falta de respuesta (Shrive, Stuart, Quan, \& Ghali, 2006). Tras un análisis exploratorio se comprobó que 
no había diferencias significativas en las puntuaciones medias y en las desviaciones estándares entre las muestras con datos imputados y la muestra con valores perdidos.

Se identificaron casos atípicos univariados, mediante el cálculo de puntajes $\mathrm{Z}$ para cada ítem, y multivariados, a través de prueba de distancia de Malahanobis y de los valores de influencia (Uriel \& Aldas, 2005). Se eliminaron cuatro casos atípicos del instrumento de prácticas parentales maternas porque presentaban puntuaciones atípicas univariadas en más de un ítem o puntuaciones atípicas uni y multivariadas. Se decidió mantener los casos que presentaban puntuaciones extremas en sólo un ítem o atípicos multivariados con valores de influencia inferiores a 1.0.

Las muestras finales quedaron conformadas por 274 y 227 casos para las escalas de procesos parentales maternos y paternos, respectivamente. Esto permite respetar el criterio mínimo de cinco casos por ítem requeridos para el AFE en todas las escalas, cumpliendo incluso el ideal de 10 casos por ítem para la AFP de la madre, o aproximándose (con un promedio de nueve casos por ítem) para la AFP del padre (Nunally \& Bernstein, 1995).

También se realizó el análisis de asimetría y curtosis para cada uno de los ítems con el objetivo de analizar el tipo de distribución de las variables. Los índices de asimetría y curtosis estuvieron dentro del rango \pm 2 que es considerado aceptable e indica que las variables se aproximan a una distribución normal (George \& Mallery, 2001).

Finalmente se inspeccionó la matriz de correlaciones de las variables para detectar problemas de multicolinealidad. A pesar de que es un requisito de la técnica de AFE que exista intercorrelación entre las variables, si éstas igualan o superan índices de .90 es probable que se debilite el análisis y se obtenga una solución factorial poco estable (Pérez \& Medrano, 2010). No se registraron en este estudio correlaciones superiores a los valores aceptados (Pérez \& Medrano, 2010).

\section{Estructura factorial}

Los índices de esfericidad de Barttlet $\left(\chi^{2}\right.$ $=1694.37 ; p<.001)$ y la medida de adecuación muestral de Kaiser Meyer-Olkin $(\mathrm{KMO}=.85)$ de las prácticas parentales paternas indicaron apropiada relación entre variables y buena adecuación muestral. En el caso de las prácticas maternas, estas medidas también resultaron adecuadas $\left(\chi^{2}=\right.$ 2231.87; $p<.001 ; \mathrm{KMO}=.84)$. La solución factorial de seis dimensiones propuesta por Vazsonyi et al. (2003) no fue satisfactoria para las prácticas parentales paternas y tampoco para las maternas, dado que los seis factores mostraban agrupación inespecífica de ítems en cuanto a su contenido teórico. Además, se evidenciaba presencia de cargas cruzadas que generaba pérdida de ítems y factores con menos de tres variables. Por lo tanto se realizó una extracción de menor cantidad de factores. La solución de cinco dimensiones fue la más adecuada a los datos de la AFP de ambos padres sin alterar su fundamentación teórica. Esta solución explicó el $48.38 \%$ de las varianzas en la AFP del padre y el $45.29 \%$ de la varianza en la escala materna.

En el caso de la AFP paterna (Tabla 1) 
Tabla 1

Matriz de patrón de la solución factorial rotada (promax) de la AFP padre.

\begin{tabular}{|c|c|c|c|c|c|}
\hline & \multicolumn{5}{|c|}{ Factor } \\
\hline & 1 & 2 & 3 & 4 & 5 \\
\hline Ítem 1 & .38 & & & & \\
\hline Ítem 4 & .44 & & & & \\
\hline Ítem 6 & .48 & & & & \\
\hline Ítem 7 & & & & & .37 \\
\hline Ítem 9 & & & & & .56 \\
\hline Ítem 10 & & & & & .81 \\
\hline Ítem 11 & & .69 & & & \\
\hline Ítem 12 & & .86 & & & \\
\hline Ítem 13 & & .67 & & & \\
\hline Ítem 14 & & .76 & & & \\
\hline Ítem 15 & .75 & & & & \\
\hline Ítem 16 & .85 & & & & \\
\hline Ítem 17 & & & .70 & & \\
\hline Ítem 18 & & & .48 & & \\
\hline Ítem 19 & & & .77 & & \\
\hline Ítem 20 & .62 & & & & \\
\hline Ítem 21 & .63 & & & & \\
\hline Ítem 22 & .55 & & & & \\
\hline Ítem 23 & & & & .59 & \\
\hline Ítem 24 & & & & .49 & \\
\hline Ítem 25 & & & & .95 & \\
\hline
\end{tabular}

fueron eliminados cuatro ítems por tener peso factorial inferior a .35. Estos ítems fueron el 2, el 3 y el 5 de la subescala cercanía, y el ítem 8 de la subescala original soporte (Anexo 1). No se registraron cargas cruzadas. El primer factor agrupó los ítems pertenecientes a las subescalas comunicación y cercanía, mientras que el segundo factor quedó constituido por los ítems de monitoreo. Los ítems de conflicto cargaron en el tercer factor y los de aprobación del grupo de pares en el cuarto. El quinto factor agrupó los ítems correspondientes a la subescala soporte. De esta manera la versión final de la AFP del padre estuvo conformada por los factores originalmente propuestos por Vazsonyi et al. (2003), aunque las dimensiones comunicación y cercanía convergi- eron en una misma dimensión.

Las correlaciones interfactoriales (Tabla 2) indicaron buena relación entre los factores, con excepción de la dimensión soporte que se asoció de manera débil a la subescala cercanía y comunicación y que no estuvo relacionada a la subescala monitoreo. Todas las correlaciones fueron positivas, exceptuando el factor conflicto que se asoció de manera negativa a todas las restantes dimensiones.

Tabla 2

Correlaciones interfactoriales de la AFP padre.

\begin{tabular}{lccccc}
\hline Factor & $\mathbf{1}$ & $\mathbf{2}$ & $\mathbf{3}$ & $\mathbf{4}$ & $\mathbf{5}$ \\
\hline $\begin{array}{l}\text { 1. Cercanía y } \\
\text { comunicación }\end{array}$ & 1 & $.55^{* * *}$ & $-.32^{* * *}$ & $.37^{* * *}$ & $.17^{*}$ \\
2. Monitoreo & & 1 & $.23^{* *}$ & $.32^{* * *}$ & .12 \\
3. Conflicto & & & 1 & $-.23^{* *}$ & $-.39^{* * *}$ \\
$\begin{array}{l}\text { 4. Aprobación } \\
\text { pares }\end{array}$ & & & 1 & $.28^{* * *}$ \\
5. Soporte & & & & \\
Nota. ${ }^{*} p<.05 ; * * *$ & $p<.001$. & & & 1 \\
\hline
\end{tabular}

En relación a la AFP de la madre (Tabla 3) fue necesario eliminar dos ítems por no alcanzar el peso factorial mínimo requerido (.35): el ítem 3 de la subescala cercanía y el ítem 8 originalmente perteneciente a la subescala soporte (Anexo 2). Se evidenciaron cargas cruzadas en el ítem 13 (monitoreo) y en el ítem 15 (comunicación). No obstante, se decidió mantener los ítems debido a que en ambos casos las diferencias entre las cargas factoriales del factor principal y del secundario fueron superiores a .10. A su vez los ítems cargaban con mayor peso en los factores esperados de acuerdo con la teoría de base. Las prácticas parentales están altamente relacionadas entre sí y no es extraño que una misma conducta de los padres pueda relacionarse con diferentes dimensiones parentales. Los ítems correspondientes a comunicación saturaron todos en el primer factor, aunque también lo hizo el ítem 1 de la subescala original cercanía. El segundo factor estuvo conformado por los ítems de aprobación del grupo de pares y por los ítems 9 y 10 de la dimensión soporte (recodificado inversamente como en la versión original). De esta manera quedó con- 
formado por cinco ítems que refieren no sólo a la aprobación de los pares sino al soporte y al apoyo en un sentido más general, por lo que fue llamado aprobación. El tercer factor agrupó los ítems de conflicto y el ítem 7 correspondiente originalmente al factor soporte formulado en un sentido negativo, es decir como falta de soporte. En el cuarto factor saturaron los ítems de la dimensión cercanía y finalmente en el quinto factor se agruparon los ítems de monitoreo. De esta manera quedaron conformados los siguientes cinco factores (entre paréntesis número de ítems): comunicación (6); aprobación (5); conflicto (4); monitoreo (4) y cercanía(4).

Tabla 3

Matriz de patrón de solución factorial rotada (promax) de la AFP madre.

\begin{tabular}{|c|c|c|c|c|c|}
\hline & \multicolumn{5}{|c|}{ Factor } \\
\hline & 1 & 2 & 3 & 4 & 5 \\
\hline Ítem 1 & .42 & & & & \\
\hline Ítem 2 & & & & .57 & \\
\hline Ítem 4 & & & & .35 & \\
\hline Ítem 5 & & & & .63 & \\
\hline Ítem 6 & & & & .70 & \\
\hline Ítem 7 & & & .51 & & \\
\hline Ítem 9 & & -.40 & & & \\
\hline Ítem 10 & & -.51 & & & \\
\hline Ítem 11 & & & & & .38 \\
\hline Ítem 12 & & & & & .78 \\
\hline Ítem 13 & .37 & & & & .49 \\
\hline Ítem 14 & & & & & .75 \\
\hline Ítem 15 & .42 & & & .31 & \\
\hline Ítem 16 & .49 & & & & \\
\hline Ítem 17 & & & .63 & & \\
\hline Ítem 18 & & & .69 & & \\
\hline Ítem 19 & & & .77 & & \\
\hline Ítem 20 & .78 & & & & \\
\hline Ítem 21 & .56 & & & & \\
\hline Ítem 22 & .63 & & & & \\
\hline Ítem 23 & & .76 & & & \\
\hline Ítem 24 & & .51 & & & \\
\hline Ítem 25 & & .80 & & & \\
\hline
\end{tabular}

Tabla 4

Correlaciones interfactoriales de la AFP de la madre.

\begin{tabular}{lccccc}
\hline Factor & $\mathbf{1}$ & $\mathbf{2}$ & $\mathbf{3}$ & $\mathbf{4}$ & $\mathbf{5}$ \\
\hline $\begin{array}{l}\text { 1. Cercanía y } \\
\text { comunicación }\end{array}$ & 1 & $.40^{* * *}$ & $-.16^{* *}$ & $.53^{* * *}$ & $.51^{* * *}$ \\
2. Monitoreo & & 1 & $-.24^{* *}$ & $.37^{* *}$ & $.32^{* *}$ \\
3. Conflicto & & 1 & $-.33^{* *}$ & -.10 \\
4. Aprobación & & & 1 & $.39^{* *}$ \\
pares & & & & \\
5. Soporte & & & & 1 \\
\hline Nota. ${ }^{*} p<.05 ; * * * p<.001$ & & &
\end{tabular}

Todas las dimensiones estuvieron significativamente correlacionadas, excepto por el monitoreo, que no se asoció al conflicto. Tal como sucedió en el caso de la escala paterna, las asociaciones del factor conflicto con los restantes factores fueron negativas (Tabla 4). En la Tabla 5 se exponen las puntuaciones medias y desviaciones típicas de cada práctica parental para la muestra total y para la muestra dividida según el género de los adolescentes. Se llevó a cabo una prueba de diferencias de puntuaciones medias a través del estadístico $t$ de Student, empleando como variable de agrupación el género de los jóvenes (Tabla 5). En relación con las prácticas parentales del padre, sólo el monitoreo $\left(t_{(218)}=3.79 ; p<.001\right)$ fue diferente entre mujeres y varones, aunque la diferencia respecto al conflicto $\left(t_{(218)}=1.93 ; p=\right.$ $.055)$, resultó marginalmente significativa, siendo más elevadas las puntuaciones en el caso de las mujeres. Las puntuaciones en las subescalas de prácticas parentales maternas de monitoreo $\left(t_{(264)}\right.$ $=6.64 ; p<.001)$, aprobación $\left(t_{(264)}=3.29 ; p=\right.$ $.001)$, conflicto $\left(t_{(264)}=2.26 ; p=.025\right)$ y comunicación $\left(t_{(264)}=4.42 ; p<.001\right)$ resultaron significativamente más altas en las mujeres. No hubo diferencias entre los grupos en el nivel de cercanía con la madre $\left(t_{(264)}=.53 ; p=.594\right)$.

Tabla 5

Estadísticos descriptivos de las prácticas parentales de pa- 
dre y madre.

\section{Muestra total Mujeres Varon- \\ es \\ M (DE) MI M (DE) M (DE)}

Prácticas parentales pa-

ternas

\begin{tabular}{|c|c|c|c|c|}
\hline Monitoreo & $\begin{array}{r}10.4 \\
(4.0)\end{array}$ & 2.6 & $11.6(4.1)$ & $9.5(3.8)$ \\
\hline Conflicto & $5.4(3.2)$ & 1.8 & $5.9(3.1)$ & $5.0(3.2)$ \\
\hline $\begin{array}{l}\text { Cercanía y } \\
\text { comunicación }\end{array}$ & $\begin{array}{l}18.8 \\
(7.1)\end{array}$ & 2.4 & $19.1(7.0)$ & $\begin{array}{l}18.6 \\
(7.2)\end{array}$ \\
\hline $\begin{array}{l}\text { Aprobación } \\
\text { pares }\end{array}$ & $8.0(2.9)$ & 2.7 & $8.0(3.1)$ & $7.9(2.7)$ \\
\hline Soporte & $6.5(3.0)$ & 2.2 & $7.0(3.1)$ & $6.2(3.0)$ \\
\hline $\begin{array}{l}\text { Prácticas pa- } \\
\text { rentales ma- } \\
\text { ternas }\end{array}$ & & & & \\
\hline Monitoreo & $\begin{array}{l}11.8 \\
(3.3)\end{array}$ & 3.0 & $13.3(2.9)$ & $\begin{array}{l}10.8 \\
(3.1)\end{array}$ \\
\hline Conflicto & $7.2(3.9)$ & 1.8 & $7.8(3.9)$ & $6.7(3.9)$ \\
\hline $\begin{array}{l}\text { C o m u n i - } \\
\text { cación }\end{array}$ & $\begin{array}{l}16.1 \\
(5.2)\end{array}$ & 2.7 & $17.8(4.9)$ & $\begin{array}{l}15.0 \\
(5.2)\end{array}$ \\
\hline Aprobación & $\begin{array}{r}12.3 \\
(4.5)\end{array}$ & 2.5 & $13.4(4.4)$ & $\begin{array}{r}11.6 \\
(4.6)\end{array}$ \\
\hline Cercanía & $\begin{array}{l}12.5 \\
(2.7)\end{array}$ & 3.1 & $12.6(2.9)$ & $\begin{array}{l}12.4 \\
(2.6)\end{array}$ \\
\hline
\end{tabular}

Nota. $\mathrm{MI}=$ Media por ítem.

\section{Consistencia interna}

Los índices de fiabilidad de las subescalas fueron de aceptables $(\alpha .=70)$ a buenos $(\alpha=$ .84) para ambos padres (Tabla 6), aunque el factor soporte de la escala paterna evidenció una consistencia interna moderada $(\alpha=.64)$.

Tabla 6

Consistencia interna de las subescalas de la AFP para padre y madre.

\begin{tabular}{lllll}
\hline & Padre & \multicolumn{3}{c}{ Madre } \\
Escala & $\alpha$ & N $^{\circ}$ ítems & $\alpha$ & $\mathbf{N}^{\circ}$ ítems \\
\hline Monitoreo & .83 & 4 & .74 & 4 \\
Conflicto & .73 & 3 & .73 & 4 \\
Cercanía y comu- & .84 & 8 & & \\
nicación & & & & \\
Aprobación pares & .70 & 3 & & \\
Soporte & .64 & 6 & & \\
Cercanía & & & .70 & 4 \\
Comunicación & & & .80 & 6 \\
Aprobación & & & .76 & 5 \\
\hline
\end{tabular}

Tabla 7

Correlaciones entre las escalas de prácticas parentales y una escala de conducta antinormativa.

\begin{tabular}{|c|c|c|c|c|c|c|}
\hline & \multicolumn{6}{|c|}{ Subescalas NDS } \\
\hline & Vandalismo & $\begin{array}{l}\text { Consumo } \\
\text { de alcohol }\end{array}$ & $\begin{array}{c}\text { Consumo de } \\
\text { drogas }\end{array}$ & Robo & Agresiones físicas & $\begin{array}{c}\text { Escala } \\
\text { Total }\end{array}$ \\
\hline \multicolumn{7}{|l|}{ Factores AFP padre } \\
\hline Cercanía y comunicación & $-.16^{*}$ & $-.19^{* *}$ & $-.20^{* *}$ & -.08 & -.10 & $-.20^{* *}$ \\
\hline Soporte & $-.21^{* *}$ & -.09 & -.11 & -.12 & $-.15^{*}$ & $-.16^{*}$ \\
\hline Monitoreo & $-.30^{* * *}$ & $-.27^{* * *}$ & $-.32^{* * *}$ & $-.20^{* *}$ & $-.23^{* *}$ & $.34^{* * *}$ \\
\hline Conflicto & .12 & .11 & -.06 & -.03 & .01 & .04 \\
\hline Aprobación pares & $-.17^{*}$ & -.08 & $-.24^{* * *}$ & $-.20^{* *}$ & $-.28^{* * *}$ & $-.24^{* * *}$ \\
\hline \multicolumn{7}{|l|}{ Factores AFP madre } \\
\hline Cercanía & -.10 & $-.14^{*}$ & $-.14^{*}$ & -.09 & $-.14^{*}$ & $-.15^{*}$ \\
\hline Comunicación & $-.29^{* *}$ & $-.28^{* *}$ & $-.32^{* *}$ & $-.21^{* *}$ & $-.24^{* *}$ & $-.34^{* *}$ \\
\hline Monitoreo & $-.28^{* *}$ & $-.29^{* *}$ & $-.38^{* *}$ & $-.31^{* *}$ & $-.31^{* *}$ & $-.39^{* *}$ \\
\hline Conflicto & .09 & .08 & -.02 & -0.7 & -.03 & .02 \\
\hline Aprobación & $-.28^{* *}$ & $-.15^{*}$ & $-.31^{* *}$ & $-.36^{* *}$ & $-.31^{* *}$ & $-.33^{* *}$ \\
\hline
\end{tabular}


En la Tabla 7 se reportan los resultados de los análisis de validez de criterio. Se presentaron numerosas correlaciones significativas entre las medidas de conducta antinormativa y las prácticas parentales. Estas correlaciones fueron en el sentido esperado, es decir que aquellas prácticas parentales que fomentan la comunicación, el monitoreo y la aprobación se asociaron con una reducción en las puntuaciones de la NDS. No obstante, los niveles de conflicto entre padres e hijos no parecen incidir en la conducta desviada de los adolescentes o lo hacen de manera parcial. Esto se evidencia tanto en el caso del padre como de la madre. Sin embargo, las prácticas de esta última reflejaron asociaciones más estrechas con la conducta antinormativa que las del padre.

\section{Discusión}

El presente trabajo se propuso estudiar las propiedades psicométricas de la escala Adolescent Family Process en adolescentes de Córdoba, una herramienta que cuenta con la particularidad de ser sistemáticamente utilizada en Criminología para el estudio de la relación entre prácticas parentales y conducta antinormativa en adolescentes. Se evaluó su estructura factorial a través de un análisis factorial exploratorio y se analizó su consistencia interna mediante el estadístico alfa de Cronbach. También se valoró su validez de criterio a través de su asociación con diferentes tipos de conducta antinormativa medidos con la NDS (Vazsonyi et al., 2001) adaptada a nuestro medio (Garrido et al., 2015).
Los análisis factoriales aquí reportados no soportan la dimensionalidad propuesta por Vazsonyi et al. (2003). Cuando se analizó la estructura interna de la AFP extrayendo seis factores, la solución factorial arrojaba elevadas cargas cruzadas y una distribución de los ítems de difícil interpretación teórica, por tanto se probaron soluciones factoriales de menor cantidad de factores. Tanto en el caso del padre como en el de la madre la solución que mejor se ajusta a los datos conservando, en líneas generales, la justificación teórica en la que está basada la escala se compone de cinco factores. No obstante, estos no se comportan de la misma manera en ambos padres. Las dimensiones de comunicación, aprobación del grupo de pares, conflicto y monitoreo parecen responder a lo propuesto por los autores originalmente, cargando todos sus ítems de manera clara en el factor teórico correspondiente. Sin embargo las dimensiones de soporte y de cercanía no funcionan de la misma manera en los padres. Mientras en la AFP paterna las subescalas de cercanía y comunicación parecen referenciar un mismo constructo, en la madre funcionan como dos dimensiones diferentes. Estas dos prácticas parentales conformarían lo que Steinberg \& Silk (2002) llaman armonía y no es extraño que estén altamente relacionadas, fusionándose incluso para el caso del padre en una misma dimensión. A su vez, si bien en la AFP de la madre se agrupan como dos factores distintos, un ítem originalmente perteneciente a cercanía carga en comunicación y la correlación entre estos factores es la más robusta. En el sentido opuesto, la dimensión de soporte, que en el padre se manifiesta como un factor diferenciado, en la escala de prácticas maternas muestra sus ítems subsumidos por otras subescalas (conflicto y aprobación del grupo de pares) en congruencia con su contenido teórico. En algunos estudios previos en inglés (Vazsonyi 
et al., 2003) y en español (Molinero, 2006) se observa que la dimensión de soporte es la que presenta los índices más bajos de confiabilidad, tanto para la madre como para el padre. Si bien esta subescala se conservó en la AFP paterna del presente estudio, esta arrojó índices de confiabilidad por debajo de las restantes subescalas. Futuras líneas de investigación que permitan una mayor profundización teórica sobre estas diferencias en las prácticas maternas y paternas serán beneficiosas.

Fue necesario eliminar algunos ítems por no alcanzar el peso factorial mínimo requerido. Los dos ítems que evidenciaron cargas cruzadas en la AFP materna se mantuvieron debido a la relevancia teórica del factor al que pertenecen y a las consecuencias prácticas y teóricas de su eliminación, considerando además que sus cargas principales se corresponden a lo propuesto por los autores en la escala original. No obstante, debido a que conservar ítems multidimensionales puede resultar problemático, estudios futuros deberán indagar si una modificación en estos ítems o el anexo de ítems nuevos al factor correspondiente resulta en un beneficio para la escala.

En la adaptación de la escala al castellano en estudiantes peruanos (Molinero, 2006) ya se evidencia una fuerte presencia de cargas secundarias. Si bien en la versión final del presente estudio tres de las subescalas de la AFP paterna quedaron compuestas solamente por tres ítems, la estructura factorial resultante responde a la base teórica que lo sustenta (Vazsonyi et al., 2003). El criterio teórico es importante al igual que los otros criterios para la conservación de los ítems (Lloret-Segura et al., 2014). Se probaron soluciones factoriales de menor cantidad de di- mensiones que no resultaron apropiadas para su interpretación (Pérez \& Medrano, 2010). En consonancia con esto, algunos autores postulan que es preferible conservar un mayor número de factores que eliminar factores potencialmente necesarios, lo cual llevaría a una pérdida de información valiosa (Fava, Velicer, \& Prochaska, 1995; Zwick \& Velicer, 1986). Esta solución factorial de cinco dimensiones no se corresponde a lo encontrado por Molinero (2006) con adolescentes de Perú, quien aporta evidencia en favor de la estructura de seis subescalas propuesta originalmente por Vazsonyi et al. (2003). No se descarta que las diferencias en las muestras empleadas y en los criterios utilizados para el desarrollo de los análisis puedan explicar los resultados disímiles.

Los índices de consistencia interna de las subescalas tanto maternas como paternas fueron de aceptables a buenos, ligeramente inferiores a los reportados en estudios previos en inglés (Vazsonyi \& Belliston, 2007; Vazsonyi et al., 2003), pero congruentes e incluso superiores a lo encontrado en otros trabajos que emplean la versión en español (Ascenzo-Bravo de Rueda, 2012; Molinero, 2006).

Los análisis de validez de criterio indicaron que el monitoreo, la comunicación y la aprobación (general en el caso de la madre, y específicamente relacionada a los pares en el caso del padre) son las prácticas más vinculadas a los distintos tipos de conducta antinormativa, mientras que el conflicto, el soporte y la cercanía no están asociados a la conducta antisocial de los adolescentes, o lo están sólo de manera parcial. La asociación de las prácticas parentales con la conducta antisocial de los hijos sigue el mismo patrón para ambos padres. No obstante, la fuerza 
de las correlaciones es mayor en el caso de las prácticas parentales maternas, las cuales, a su vez, presentan en la mayoría de los factores puntuaciones medias por ítem superiores a las paternas. Pocos estudios han valorado la influencia de la parentalidad en la conducta adolescente discriminando entre los sexos de los padres (Williams, 2005). Algunos trabajos han encontrado que las prácticas maternas influyen más que las paternas en la conducta agresiva adolescente (Tur-Porcar, Mestre, Samper, \& Malonda, 2012). No obstante, otros estudios no soportan estos hallazgos (Mestre et al., 2004).

Si se tiene en cuenta el género de los adolescentes, las mujeres reportan más conflicto, comunicación y aprobación por parte de sus madres que los varones, mientras que no hay diferencias entre los grupos cuando esas prácticas parentales corresponden al padre. Las mujeres también indican ser más supervisadas por ambos padres, siendo el monitoreo la única práctica parental paterna que se diferencia en adolescentes mujeres y varones.

En líneas generales, las herramientas analizadas han demostrado ser válidas y confiables en adolescentes de Córdoba. No obstante, es necesario remarcar algunas limitaciones. En primer lugar, sería deseable que estudios futuros comprueben la estructura factorial de las escalas en muestras de infractores y no infractores de manera independiente, para evaluar si se corresponde a lo encontrado en el análisis de la muestra total. Esto no pudo ser llevado a cabo en este trabajo debido a que no se contaba con la cantidad de casos necesaria en cada grupo. Futuros estudios también podrían contribuir a lograr un mayor entendimiento de la influencia diferen- cial de estas prácticas en función del género de los adolescentes evaluando la invarianza factorial de la escala en mujeres y varones. El tamaño muestral limitado tampoco permitió realizar esta valoración en el presente estudio.

Pese a las limitaciones expuestas, los resultados que aquí se presentan constituyen uno de los primeros intentos de adaptar al contexto local instrumentos tradicionalmente empleados en la literatura criminológica para evaluar prácticas parentales, una variable sistemáticamente asociada al delito en jóvenes. Es de relevancia también el esfuerzo por incorporar a sujetos pertenecientes a poblaciones consideradas representativas del comportamiento antisocial (menores infractores) (Mathews, Youman, Stuewig, \& Tangney, 2007).

Dada la poca representación de nuestro medio en la literatura científica criminológica, se considera necesario conducir estudios que permitan derivar recomendaciones con fines preventivos adaptadas al contexto local y para ello es una tarea relevante proveer a los profesionales de instrumentos y herramientas de medición válidos y confiables. Este estudio se enmarca en una línea de trabajo que resalta la importancia de una aproximación empírica al delito y que intenta dotar de validez ecológica a las investigaciones, a través del estudio de poblaciones específicas y representativas del comportamiento criminal. 


\section{Referencias}

Arbach, K., Santuoro, S., Lumello, A., \& Garrido, S. (Agosto de 2013). Evaluación de la conducta antinormativa durante la adolescencia. En XIV Reunión Nacional y III Encuentro Internacional de la Asociación Argentina de Ciencias del Comportamiento. Córdoba, Argentina.

Ascenzo-Bravo de Rueda, F. (2012). Características asociadas a los trastornos alimenticios y percepción de la función parental en adolescentes escolares (Tesis de pregrado). Universidad Católica del Perú, Lima, Perú.

Asili-Pierucci, N., \& Pinzón-Luna, B. K. (2003). Relación entre estilos parentales, estilos de apego y bienestar psicológico. Psicología y Salud, 13(2), 215-225.

Baumrind, D. (2005). Patterns of parental authority and adolescent autonomy. New Directions for Child and Adolescent Development, 2005(108), 61-69. doi: 10.1002/cd.128

Brussino, S., \& Alderete, A. M. (2002). Inventario de pautas de crianza: Estudio de la consistencia. Evaluar, 2(1), 67-77.

Carlo, G., McGinley, M., Hayes, R., Batenhorst, C., \& Wilkinson, J. (2007). Parenting styles or practices? Parenting, sympathy, and prosocial behaviors among adolescents. The Journal of Genetic Psychology, 168(2), 147-176. doi: 10.3200/gntp.168.2.147-176

Cernkovich, S., \& Giordano, P. (1987). Family relationships and delinquency. Criminology, 25(2), 295-321. doi: 10.1111/j.17459125.1987.tb00799.x
Eichelsheim, V. I., Buist, K. L., Deković, M., Wissink, I. B., Frijns, T., Van Lier, P. A. C., ... Meeus, W. H. J. (2010). Associations among the parent-adolescent relationship, aggression and delinquency in different ethnic groups: A replication across two dutch samples. Social Psychiatry and Psychiatric Epidemiology, 45(3), 293-300. doi: 10.1007/s00127-009-0071-z

Fava, J. L., Velicer, W. F., \& Prochaska, J. O. (1995). Applying the transtheoretical model to a representative sample of smokers. $A d$ dictive Behaviors, 20(2), 189-203. doi: 10.1016/0306-4603(94)00062-х

Franco-Nerín, N., Pérez-Nieto, M. A., \& De Dios-Pérez, M. J. (2014). Relación entre los estilos de crianza parental y el desarrollo de ansiedad y conductas disruptivas en niños de 3 a 6 años. Revista de Psicología Clínica con Niños y Adolescentes, 1(2), 149156. Recuperado de https://dialnet. unirioja.es/descarga/articulo/4742071.pdf

Garrido, S., Cupani, M., \& Arbach, K. (2015). Conducta antinormativa y autocontrol: Adaptación de dos escalas de evaluación en población juvenil de Córdoba (Tesis de pregrado). Universidad Nacional de Córdoba, Córdoba, Argentina.

George, D., \& Mallery, P. (2001). SPSS for Windows step by step: A simple guide and reference. Boston, MA: Allyn and Bacon.

Haegerich, T. M., Oman, R. F., Vesely, S. K., Aspy, C. B., \& Tolma, E. L. (2014). The predictive influence of family and neighborhood assets on fighting and weapon carrying from mid- to late adolescence. Prevention Science, 15(4), 473-484. doi: 10.1007/ s11121-013-0400z 
Hair, J. F., Anderson, R. E., Tatham, R. L., \& Black, W. (1999). Análisis multivariante (5 ${ }^{\mathrm{a}}$ Edición). Madrid: Prentice Hall.

Hernández-Guzmán, L., Montesinos, M. G., Bermúdez-Ornelas, G., Freyre, M. A., \& Alcázar-Olán, R. J. (2013). Parental practices scale for children. Revista Colombiana de Psicología, 22(1), 151-161.

Hoeve, M., Dubas, J. S., Eichelsheim, V. I., Van der Laan, P. H., Smeenk, W., \& Gerris, J. (2009). The relationship between parenting and delinquency: A meta-analysis. Journal of Abnormal Child Psychology, 37(6), 749775. doi: 10.1007/s10802-009-9310-8

Hoeve, M., Stams, G. J., Van der Put, C., Dubas, J. S., Van der Laan, P. H., \& Gerris, J. (2012). A meta-analysis of attachment to parents and delinquency. Journal of Abnormal Child Psychology, 40(5), 771-785. doi: 10.1007/s10802-011-9608-1

IBM Corp. (2013) SPSS Statistics for Windows, Version 22.0. Armonk, NY: IBM Corp.

Lloret-Segura, S., Ferreres-Traver, A., Hernández-Baeza, A., \& Tomás-Marco, I. (2014). $\mathrm{El}$ análisis factorial exploratorio de los ítems: Una guía práctica, revisada y actualizada. Anales de Psicología, 30(3), 11511169. doi: 10.6018/analesps.30.3.199361

Loeber, R. (1990). Development and risk factors of juvenile antisocial behavior and delinquency. Clinical Psychology Review, 10, 1-41. doi: 10.1016/0272-7358(90)90105-J

Maccoby, E. E., \& Martin, J. A. (1983). Socialization in the context of the family: parent-child interaction. En E. M. Hetherington \& P. H. Mussen (Eds.), Handbook of Child Psychology, Vol. IV: Socialization, Personality and Social Development (4a ed., pp. 1-101). Nueva York: Wiley.

Mathews, J., Youman, K., Stuewig, J., \& Tangney, J. (2007). Reliability and validity of the Brief Self-Control Scale in a sample of incarcerated offenders. En Annual meeting of the American Society of Criminology. Atlanta Marriott Marquis, Atlanta, GA.

McBride-Chang, C., \& Chang, L. (1998). Adolescent-parent relations in Hong Kong: parenting styles, emotional autonomy, and school achievement. Journal of Genetic Psychology, 159(4), 421-436. doi: $10.1080 / 00221329809596162$

Mestre, V., Samper, P., \& Frías, D. (2004). Personalidad y contexto familiar como factores predictores de la disposición prosocial y antisocial de los adolescentes. Revista Latinoamericana de Psicología, 36(3), 445-457.

Metzler, C. W., Biglan, A., Ary, D. V, \& Li, F. (1998). The stability and validity of early adolescents' reports of parenting constructs. Journal of Family Psychology, 12(4), 600-619. doi: 10.1037/0893-3200.12.4.600

Milevsky, A., Schlechter, M., Netter, S., \& Keehn, D. (2007). Maternal and paternal parenting styles in adolescents: Associations with self-esteem, depression and lifesatisfaction. Journal of Child and Family Studies, 16(1), 39-47. doi: 10.1007/s10826-006-9066-5

Molinero, C. (2006). Adaptación de la escala «Adolescent Family Process» (AFP) en una muestra de adolescentes de Lima Metropolitana y Callao (Tesis de pregrado). Universidad Católica del Perú, Perú.

Nunally, J. C., \& Bernstein, I. H. (1995). Teoría Psicométrica. Mexico: McGraw-Hill. 
Oliva, A., Parra, Á., \& Arranz, E. (2008). Parenting styles and adolescent adjustment [Estilos relacionales parentales y ajuste adolescente]. Infancia y Aprendizaje, 31(1), 93-106. doi: 10.1174/021037008783487093

Ozdemir, Y., Vazsonyi, A. T., \& Cok, F. (2013). Parenting processes and aggression: The role of self-control among Turkish adolescents. Journal of Adolescence, 36(1), 65-77. doi: 10.1016/j.adolescence.2012.09.004

Pelegrina-López, S., García-Linares, M. C., \& Lendínez, J. (2002). Los estilos educativos de los padres y la competencia psicosocial de los adolescentes. Anuario de psicología, 33(1), 79-96. doi: 10.1174/021037002317417796

Pérez, E. R., \& Medrano, L. (2010). Análisis factorial exploratorio: Bases conceptuales y metodológicas. Revista Argentina de Ciencias del Comportamiento, 2(1889), 58-66.

Pérez-Alonso, P. M. (2012). La socialización parental en padres españoles con hijos de 6 a 14 años. Psicothema, 24(3), 371-376.

Petrosino, A., Derzon, J., \& Lavenberg, J. (2009). The role of the family in crime and delinquency: Evidence from prior quantitative reviews. Southwest Journal of Criminal Justice, 6(2), 108-132.

Pickering, L. E., Smith, T. A., \& Vaughn, B. E. (2005). Hellfire, home, and harm: An investigation of the interaction between religiosity, family processes, and adolescent deviant behavior (Tesis doctoral). Auburn University, Alabama, Estados Unidos.

Pickering, L. E., \& Vazsonyi, A. T. (2010). Does family process mediate the effect of religiosity on adolescent deviance? Revisit- ing the notion of spuriousness. Criminal Justice and Behavior, 37(1), 97-118. doi: 10.1177/0093854809347813

Piquero, A. R., Hawkins, J. D., Kazemian, L., Petechuk, D., \& Redondo, S. (2013). Serie especial: La transición desde la delincuencia juvenil a la delincuencia adulta. Revista Española de Investigación Criminológica, l(11), 1-40.

Polo, C. (2009). Resiliencia: factores protectores en adolescentes de 14 a 16 años (Tesis de pregrado). Universidad de Aconcagua, Mendoza, Argentina. Recuperado de http://bibliotecadigital.uda.edu.ar/objetos_digitales/71/ tesis-1426-resiliencia.pdf

Pons-Diez, J., \& Berjano-Peirats, E. (1997). Analisis de los estilos parentales de socialización asociados al abuso de alcohol en adolescentes. Psicothema, 9(3), 609-617.

Rechea, C. (2008). Conductas antisociales y delictivas de los jóvenes en España. España. Recuperado de https://previa.uclm.es/centro/ criminologia/pdf/informes/16_2008.pdf

Richaud, M. (2007). La percepción de estilos de relación con su padre y madre en niños y niñas de 8 a 12 años. Revista Iberoamericana de Diagnóstico y Evaluación Psicológica, 23(1), 63-81. Recuperado de http://www.aidep.org/03_ridep/R23/R234. pdf

Roberts, M., \& Steinberg, L. (1999). Unpacking authoritative parenting: Reassessing a multidimensional construct. Journal of Marriage and Family, 61(3), 574-587. doi: $10.2307 / 353561$

Sánchez, L. C. (2014). Adaptación del indice de estilos parentales en adolescentes de 14 a 18 
años de la ciudad de Concordia (Tesis de pregrado). Universidad Católica Argentina, Facultad Teresa de Ávila, Entre Ríos, Argentina. Recuperado de http://bibliotecadigital.uca.edu.ar/greenstone/cgi-bin/library. cgi $\mathrm{a}=\mathrm{d} \& \mathrm{c}=$ tesis $\& \mathrm{~d}=$ adaptacion-indice-estilos-parentales

Santander-Montes, A., \& Ruiz-Vaquero, R. (2004). Relación entre variables cuantitativas. Cuba: ECIMED.

Shrive, F. M., Stuart, H., Quan, H., \& Ghali, W. A. (2006). Dealing with missing data in a multi-question depression scale: a comparison of imputation methods. BMC Medical Research Methodology, 6(1), 57. doi: 10.1186/1471-2288-6-57

Snider, J. B., Clements, A., \& Vazsonyi, A. T. (2004). Late adolescent perceptions of parent religiosity and parenting processes. Family Process, 43(4), 489-502. doi: 10.1111/j.15455300.2004.00036.x

Steinberg, L. (2001). We know some things: Parent - adolescent relationships in retrospect and prospect. Journal of Research on $\mathrm{Ad}$ olescence, 11(1), 1-19. doi: 10.1111/15327795.00001

Steinberg, L., \& Silk, J. S. (2002). Parenting adolescents. En M. H. Bornstein (Ed.), Handbook of parenting (pp. 103-133). Mahwah, NJ: Erlbaum. doi: 10.4324/9781410612137

Stephens, M. A. (2009). Gender differences in parenting styles and effects on the parent-child relationship (Tesis de pregrado). Texas State University, San Marcos, Texas, Estados Unidos.

Suldo, S. M., \& Huebner, E. S. (2004). The role of life satisfaction in the relationship be- tween authoritative parenting dimensions and adolescent problem behavior. Social Indicators Research, 66(1), 165-195. doi: 10.1023/B:SOCI.0000007498.62080.1e

Tabachnick, B., \& Fidell, L. (2001). Using multivariate statistics. New York: Harper and Row.

Torío López, S. (2001). Estudio socioeducativo de hábitos y tendencias de comportamiento en familias con niños de educación infantil y primaria en Asturias. Recuperado de http://digibuo.uniovi.es/dspace/handle/10651/16596

Tur-Porcar, A., Mestre, V., Samper, P., \& Malonda, E. (2012). Crianza y agresividad de los menores: ¿es diferente la influencia del padre y de la madre? Psicothema, 24(2), 284-288.

Uriel, E., \& Aldas, J. (2005). Análisis Multivariante Aplicado. España: Thomson.

Vazsonyi, A. (2003). Parent-Adolescent Relations and Problem Behaviors. Marriage and Family Review, 35(3-4), 161-187. doi: 10.1300/j002v35n03_09

Vazsonyi, A., \& Belliston, L. (2007). The family, low selfcontrol, deviance: A cross-cultural and cross-national test of Self-Control Theory. Criminal Justice and Behavior, 34(4), 505530. DOI: $10.1177 / 0093854806292299$

Vazsonyi, A., \& Flannery, D. J. (1997). Early adolescent delinquent behaviors: Associations with family and school domains. The Journal of Early Adolescence, 17(3), 271-293. doi: 10.1177/0272431697017003002

Vazsonyi, A., Hibbert, J. R., \& Blake-Snider, J. (2003). Exotic enterprise no more? Adolescent reports of family and parenting processes from youth in four countries. 
Journal of Research on Adolescence, 13(2), 129-160. doi.org/10.1111/15327795.1302001

Vazsonyi, A., Ksinan-Jiskrova, G., Ksinan, A., \& Blatny, M. (2016). An empirical test of self-control theory in Roma adolescents. Journal of Criminal Justice, 44, 66-76. doi: 10.1016/j.jcrimjus.2015.12.004

Vazsonyi, A., Pickering, L., Junger, M., \& Hessing, D. (2001). An empirical test of a general theory of crime: A fournation comparative study of self-control and the prediction of deviance. Journal of Research in Crime and Delinquency, 38(2), 91-131. doi: 10.1177/0022427801038002001

Vazsonyi, A., \& Rudi, K. (2008). Test of Self-Control Theory across different socioeconomic strata. Justice Quarterly, 25(1), 101-131. doi: 10.1080/07418820801954571

Videon, T. M. (2005). Parent-child relations and children's psychological well-being: Do dads matter? Journal of Family Issues, 26(1), 55-78. doi: 10.1177/0192513X04270262

Williams, S. K. (2005). Relationships among involvement, attachment, and behavioral problems in adolescence: Examining father's influence. The Journal of Early Adolescence, 25(2), 168-196. doi: $10.1177 / 0272431604274178$

Zwick, W. R., \& Velicer, W. F. (1986). Comparison of five rules for determining the number of components to retain. Psychological Bulletin, 99(3), 432-442. doi: 10.1037/00332909.99.3.432 